\title{
Programme d'indemnisation des victimes d'une vaccination : Justification et aperçu du programme du Québec
}

\author{
Eve Dubé1,2*, Dominique Gagnon ${ }^{1}$, Noni E MacDonald ${ }^{3}$, Shawn Harmon ${ }^{3}$, \\ Sandani Hapuhennedige ${ }^{4}$
}

\section{Résumé}

Les vaccins sont parmi les agents thérapeutiques les plus sûrs, et les effets indésirables graves se produisent rarement. Lorsqu'ils se produisent, une personne peut devoir assumer une partie ou la totalité des coûts associés à ses blessures, demander une indemnisation par voie de litige ou, si possible, demander une indemnisation dans le cadre d'un programme d'indemnisation des victimes d'une vaccination financé par le secteur public. Les programmes $d^{\prime}$ 'indemnisation des victimes d'une vaccination sont des régimes d'indemnisation " sans égard à la faute » dans le cadre desquels les gouvernements indemnisent les personnes lésées par des vaccins fabriqués adéquatement. II existe des justifications éthiques, juridiques et pratiques à l'appui de ces programmes. À l'échelle mondiale, 19 pays ont mis en œuvre des programmes d'indemnisation des victimes d'une vaccination; dans la majorité de ces pays, les vaccins ne sont pas obligatoires. Ils ont tous des processus semblables en ce qui concerne les façons d'appliquer, les preuves nécessaires et les éléments d'indemnisation. Au Canada, seule la province de Québec a un programme d'indemnisation des victimes d'une vaccination, lequel fonctionne avec succès depuis 1985. Les programmes d'indemnisation des victimes d'une vaccination soulèvent des préoccupations, notamment en ce qui a trait au coût, à la difficulté d'évaluer la causalité et à la crainte que de tels programmes puissent miner la confiance du public dans les vaccins, mais ces préoccupations peuvent être surmontées, surtout dans les pays à revenu élevé qui peuvent assumer les coûts et qui ont la capacité de gérer le programme.
Cette oeuvre est mise à la disposition selon les termes de la licence internationale Creative Commons Attribution 4.0

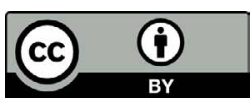

\begin{abstract}
Affiliations
1 Institut national de santé publique du Québec, QC

${ }^{2}$ Centre de recherche du $\mathrm{CHU}$ de Québec - Université Laval, QC

${ }^{3}$ Université Dalhousie, Halifax, NS

${ }^{4}$ Université de Toronto, Toronto, ON
\end{abstract}

\section{${ }^{\star}$ Correspondance : \\ eve.dube@inspq.qc.ca}

Citation proposée : Dubé E, Gagnon D, MacDonald NE, Harmon SHE, Hapuhennedige S. Programme d'indemnisation des victimes d'une vaccination : Justification et aperçu du programme du Québec. Relevé des maladies transmissibles au Canada 2020;46(9):344-8. https://doi.org/10.14745/ccdr.v46i09a09f

Mots-clés : vaccin, indemnisation, assurance sans égard à la faute, considérations éthiques

\section{Introduction}

Les vaccins sont parmi les outils de prévention les plus sûrs et les plus efficaces. Pourtant, les vaccins, comme toute intervention médicale, ne sont pas sans risque, bien qu'ils soient minimes. La plupart des manifestations cliniques indésirables suite à la vaccination sont bénignes et se résolvent rapidement et complètement (e.g. fièvre, enflure au point d'injection, éruptions cutanées, etc.). Toutefois, dans de rares cas, des effets indésirables graves peuvent se produire même si la conception, la fabrication et l'administration du vaccin sont adéquates (1). Une manifestation clinique indésirable grave suite à la vaccination est définie comme une situation qui met la vie en danger, qui nécessite une hospitalisation ou une prolongation de l'hospitalisation existante, qui entraîne une incapacité ou une incapacité persistante ou importante ou qui entraîne une anomalie congénitale (2). Le risque absolu des manifestations cliniques indésirable grave suite à la vaccination est extrêmement faible (e.g. moins d'une dose par 10 millions pour les vaccins toxoïdes contre le tétanos; de 1 à 2 doses par million pour le vaccin antigrippal inactivé) (3). Au niveau de la population, ces rares risques graves sont largement compensés par les avantages d'un taux élevé de vaccination. Toutefois, cela implique que, dans de rares cas, une personne subira des conséquences importantes au profit d'autres personnes, et qu'un tel événement peut être anticipé (même prévu), mais pas nécessairement prédit au niveau individuel (1).

Cela nous amène à nous demander quels sont les rôles et les responsabilités des pays et autorités de santé par rapport à 
ceux qui subissent une «blessure causée par une vaccination » (i.e. une manifestation clinique indésirable grave suite à la vaccination) lorsqu'ils reçoivent un vaccin recommandé par la santé publique. Halabi et Omer (3) ont déterminé trois types d'approches à l'égard des manifestations cliniques indésirables suite à la vaccination. Bien que les frais importants associés à un manifestation clinique indésirable grave suite à la vaccination soient couverts par le système de santé publique, les personnes peuvent 1) assumer elles-mêmes les coûts associés à leurs blessures, 2) demander une indemnisation à l'aide de poursuites contre des acteurs du secteur privé (i.e. les fabricants de vaccins) ou 3) chercher à obtenir une indemnisation auprès de systèmes financés par l'État ou de programmes d'indemnisation des victimes d'une vaccination (programmes d'indemnisation des victimes d'une vaccination) (3) lorsqu'il s'agit de tout coût supplémentaire.

L'objectif de cet article est de fournir une justification et un aperçu global des programmes d'indemnisation des victimes d'une vaccination et de décrire la situation au Canada et, plus précisément, au Québec. Il s'agit du septième d'une série d'articles produits par le Centre canadien de ressources et d'échange de données probantes sur la vaccination (CANVax). Ce Centre comprend un groupe de professionnels multidisciplinaires qui déterminent et créent des ressources utiles pour favoriser l'adoption de vaccins.

\section{Justification des programmes d'indemnisation des victimes d'une vaccination}

Il y a plusieurs raisons pour lesquelles les pays et territoires ont mis en œuvre des programmes d'indemnisation des victimes d'une vaccination. Comme l'ont souligné Looker et Kelly, ces programmes découlent souvent de pressions politiques et économiques, de menaces de litiges et de l'impératif d'assurer un approvisionnement continu en vaccins $(4,5)$. En général, il existe des arguments biologiques, éthiques, juridiques et pratiques à l'appui de la mise en œuvre des programmes $d$ 'indemnisation des victimes d'une vaccination (6).

- Motifs biologiques : Les vaccins sont extrêmement sécuritaires, mais la possibilité de préjudice existe dans de rares cas et a été reconnue (e.g. anaphylaxie, intussusception suite à un vaccin antirotavirus qui n'est plus utilisé)

- Motifs éthiques : La vaccination profite non seulement à la personne vaccinée, mais à toute la communauté grâce à l'immunité collective. Les principes éthiques de solidarité, de réciprocité, d'équité et de justice soutiennent tous la mise en œuvre de mesures visant à indemniser les quelques personnes qui seront lésées par les vaccins. Ces arguments sont plus forts dans les administrations où les gouvernements utilisent des politiques obligatoires pour assurer une vaccination généralisée

- Motifs juridiques : Sur le plan juridique, nous avons créé une société fondée sur les droits où l'intégrité physique de chacun est, dans une certaine mesure, garantie et où les atteintes à cette intégrité donnent lieu à des demandes de réparation justifiées

- Motifs pratiques : Les litiges de responsabilité civile liés aux manifestations cliniques indésirables suite à la vaccination sont coûteux et incertains, et l'exposition à cette incertitude et à cette responsabilité potentielle peut décourager les fabricants de produire des vaccins. Les programmes $d$ 'indemnisation des victimes d'une vaccination éliminent I'incertitude des litiges pour les fabricants et assurent la sécurité de l'approvisionnement en vaccins. Ils contribuent également à créer un environnement propice à l'innovation en matière de vaccins

\section{Aperçu global}

Un examen récent a montré que des programmes d'indemnisation ont été mis en œuvre dans 19 administrations dans le monde. Il est intéressant de noter que pour douze de ces administrations qui ont de tels programmes, la vaccination n'est pas obligatoire (tableau 1) (4).

\section{Tableau 1 : Administrations ayant des programmes d'indemnisation des victimes de vaccination (y compris l'année d'introduction)}

\begin{tabular}{|l|l|}
\hline \multicolumn{1}{|c|}{ Vaccination non obligatoire } & \multicolumn{1}{|c|}{ Vaccination obligatoire } \\
\hline Allemagne (1961) & États-Unis (1988) \\
Autriche (1973) & France (1963) \\
Danemark (1972) & Hongrie (2005) \\
Finlande (1984) & Italie (1992) \\
Islande (2001) & République de Corée (1994) \\
Japon (1970) & Slovénie (2004) \\
Nouvelle-Zélande (1974) & Taïwan (1988) \\
Norvège (1995) & \\
Québec (1985) & \\
Royaume-Uni (1979) & \\
Suède (1978) & \\
Suisse (1970) & Sources: Looker et Kelly, 2011 (1); Attwell et al., 2019 (4)
\end{tabular}

Les programmes d'indemnisation des victimes d'une vaccination sont des régimes d'indemnisation "sans égard à la faute " dans le cadre desquels les gouvernements fédéral et provinciaux indemnisent les personnes qui subissent un préjudice en raison de vaccins bien fabriqués (3). La manière dont ces programmes sont administrés, les personnes éligibles et les vaccins couverts, le processus de décision pour l'administration et la manière dont les fonds sont obtenus et répartis varient considérablement (5). 
Tableau 2 : Éléments communs des programmes d'indemnisation des victimes d'une vaccination

\begin{tabular}{|c|c|}
\hline Élément & Commentaire \\
\hline Administration & $\begin{array}{l}\text { La plupart des programmes d'indemnisation } \\
\text { sont mis en œuvre et administrés par le } \\
\text { gouvernement à l'échelle nationale ou } \\
\text { infranationale }\end{array}$ \\
\hline Financement & $\begin{array}{l}\text { Trésors nationaux, étatiques ou municipaux } \\
\text { Redevance des fabricants } \\
\text { Taxe sur les vaccins }\end{array}$ \\
\hline Admissibilité & $\begin{array}{l}\text { Seuls les vaccins obligatoires } \\
\text { Seulement les vaccins recommandés par la } \\
\text { santé publique } \\
\text { Tous les vaccins homologués } \\
\text { Seuls les vaccins dont on croit qu'ils } \\
\text { présentent un risque connexe (e.g. tableau } \\
\text { des blessures par vaccin) }\end{array}$ \\
\hline Processus & $\begin{array}{l}\text { Le processus est semblable dans la plupart } \\
\text { des secteurs de compétence : } \\
\text { - Critères de seuil de blessure ou } \\
\text { d'invalidité à respecter avant de présenter } \\
\text { une demande d'indemnisation } \\
\text { - Révision initiale par un organisme } \\
\text { administratif des décisions initiales } \\
\text { d'admissibilité et d'indemnisation } \\
\text { - Révision par un comité externe d'examen } \\
\text { si une demande est jugée complexe ou } \\
\text { litigieuse } \\
\text { - Processus d'appel officiel pour les } \\
\text { demandeurs } \\
\text { - Priorisation du règlement rapide des } \\
\text { réclamations }\end{array}$ \\
\hline Norme de preuve & $\begin{array}{l}\text { "Prépondérance des probabilités " (i.e. plus } \\
\text { de preuves qu'aucune preuve qu'un vaccin a } \\
\text { causé la blessure) } \\
\text { Cause probable } \\
\text { «Probabilité prépondérante» }\end{array}$ \\
\hline $\begin{array}{l}\text { Éléments de } \\
\text { l'indemnisation }\end{array}$ & $\begin{array}{l}\text { Montant forfaitaire ou remboursement } \\
\text { proportionnel à la gravité de la lésion causée } \\
\text { par le vaccin, notamment : } \\
\text { - Frais médicaux non remboursés } \\
\text { - Pension d'invalidité } \\
\text { - Perte non économique, notamment la } \\
\text { douleur et la souffrance } \\
\text { - Prestations de décès } \\
\text { - Indemnisation à la famille } \\
\text { - Frais juridiques raisonnables (au } \\
\text { Royaume-Uni, autant pour les demandeurs } \\
\text { dont la demande a été acceptée que ceux } \\
\text { dont la demande a été rejetée) }\end{array}$ \\
\hline Droit de litige & $\begin{array}{l}\text { Dans la plupart des pays, les demandeurs } \\
\text { peuvent demander des dommages-intérêts } \\
\text { devant les tribunaux ou une indemnisation } \\
\text { dans le cadre du programme, mais pas les } \\
\text { deux } \\
\text { D'autres pays rajustent les paiements } \\
\text { d'indemnisation si les dommages ont été } \\
\text { reçus par les tribunaux }\end{array}$ \\
\hline
\end{tabular}

Source : Looker et Kelly, 2011 (1)
Looker et Kelly (1) ont effectué un examen approfondi des éléments communs du programme (tableau 2).

\section{La situation au Canada}

Au Canada, à l'exception du Québec, tous les coûts importants des soins de santé découlant d'une blessure causée par une vaccination sont couverts par le système de santé publique. En cas d'invalidité, le soutien proviendrait probablement des revenus $d$ 'invalidité des personnes couvertes. Le seul autre moyen $d^{\prime}$ indemnisation est le recours aux tribunaux.

Il y a une compréhension limitée du nombre et de la portée des poursuites liées aux blessures causées par une vaccination au Canada (5). Nous savons que très peu de causes aboutissent devant les tribunaux, et elles sont souvent liées à des questions de procédure, et non au bien-fondé des causea (i.e. demandes d'attestation à titre de recours collectif, demandes de radiation de revendications, etc.) (7). Une exception est l'affaire Morgan c. Ville de Toronto (8), où la demanderesse a poursuivi la Ville de Toronto pour dommages-intérêts découlant du syndrome de fatigue chronique, qui, selon elle, découlait de l'administration négligente du vaccin contre I'hépatite B par la Ville en 1994. En rejetant la demande, la Cour a conclu que, bien que la norme de divulgation des risques soit très élevée, tous les soupçons de risque ne constituent pas un risque " connu " ou " important ", et que la Ville n'a pas enfreint la norme en omettant d'avertir le demandeur des effets possibles qui ne l'étaient pas, au moment de l'inoculation, considéré comme matériau. Il est à noter que de nombreux autres cas sont tranchés par des organismes comme les tribunaux d'indemnisation des accidents du travail et les tribunaux d'appel de la sécurité professionnelle et de l'assurance contre les accidents du travail, mais aucune enquête exhaustive sur les résultats de ces cas n'a été menée.

\section{Programme d'indemnisation des victimes d'une vaccination du Québec}

La description suivante provient du site Web du ministère de la Santé du Québec (https://www.msss.gouv.qc.ca/fr/). En 1979, une fillette de cinq ans, Nathalie Lapierre, a contracté une encéphalite virale peu de temps après avoir été vaccinée contre la rougeole, et elle a été gravement handicapée. Ses parents ont intenté une action en dommages-intérêts contre le gouvernement du Québec, notamment en matière de tutorat. Dans l'affaire Québec (Procureur général) c. Lapierre (9), la Cour d'appel du Québec a conclu que, bien qu'il y ait un lien de causalité entre la vaccination et la blessure, la faute n'incombait pas à la province (ou à l'infirmière qui administre la vaccination) et il n'y avait aucune obligation en vertu de la législation du Québec d'indemniser en l'absence de faute. En rejetant l'appel de l'affaire Lapierre, la Cour d'appel et la Cour suprême du Canada ont toutes deux fait observer qu'une « obligation indépendante de toute faute dans des circonstances telles celles 
du cas présent serait une excellente chose, mais notre droit actuel ne la prévoit pas ». Par conséquent, peut-être pour des raisons politiques ou en reconnaissance des exigences de la justice sociale, le gouvernement du Québec a fourni un certain soutien à la famille dans cette affaire. Par la suite, en 1985, il a introduit son programme d'indemnisation des victimes d'une vaccination, qui était fondé sur une nouvelle section de la Loi sur la protection de la santé publique. Un règlement propre à ce programme a été adopté en novembre 1987, et les premières demandes d'indemnisation ont été déposées l'année suivante.

Le principe du programme d'indemnisation des victimes d'une vaccination est que le ministre de la Santé et des Services sociaux du Québec doit indemniser toute personne blessée à la suite d'une vaccination volontaire au moyen d'un vaccin ou d'immunoglobulines contre une maladie ou une infection identifiée dans le règlement, ou contre tout vaccin obligatoire ou imposé. La vaccination doit avoir eu lieu au Québec et le formulaire de demande doit être rempli dans les trois ans suivant la blessure. La demande est examinée par un comité externe d'experts en vaccinologie qui 1) fait des recommandations au ministre sur l'existence ou l'absence d'un lien de causalité entre la blessure subie et la vaccination et 2) évalue, s'il y a lieu, le pourcentage d'atteinte permanente à l'intégrité physique ou mentale de la victime, et d'autres éléments requis concernant l'indemnisation.

Le ministre rend ensuite une décision. Si la demande est rejetée, le demandeur est informé et dispose de 60 jours pour interjeter appel. Si la demande est acceptée, le montant de l'indemnisation est déterminé en fonction des gains et des frais médicaux. Le ministre a conclu une entente avec la Société de l'assurance automobile du Québec selon laquelle la Société calcule et verse la compensation dans les cas où la décision est favorable. Les montants sont calculés conformément aux règles et règlements prescrits dans la Loi sur l'assurance automobile et sont identiques à ceux accordés en cas d'accident d'automobile.

En date du $1^{\text {er }}$ avril 2018, 228 demandes avaient été présentées et 187 répondaient aux critères d'admissibilité aux fins

d'évaluation. De ces 187 cas, 43 demandes ont été acceptées, ce qui a donné lieu à une indemnisation de 5,49 millions de dollars. II y a habituellement entre trois et cinq demandes par année, mais entre 2009 et 2012, il y a eu respectivement 11, 16, 9 et 28 demandes. En 2009 à 2010, 5,7 millions de Québécois ont reçu le vaccin contre la grippe $A(H 1 N 1)$, et cette augmentation du taux de vaccination pourrait expliquer l'augmentation des demandes observées au cours des trois années suivant les campagnes de vaccination de masse (10).

\section{Préoccupations concernant les programmes d'indemnisation des victimes d'une vaccination}

Les arguments contre les programmes d'indemnisation des victimes d'une vaccination sont souvent fondés sur les coûts de ces programmes, les difficultés liées à l'évaluation de la causalité (i.e. déterminer s'il y a un lien de causalité entre un vaccin et une blessure particulière), et la crainte que ces programmes puissent diminuer la confiance du public dans les vaccins et alimentent les mouvements anti-vaccination (4).

Certaines de ces préoccupations concernant les programmes $d$ 'indemnisation des victimes d'une vaccination ont été prises en compte. L'expérience des 19 pays et territoires où de tels programmes ont été mis en œuvre indique que les coûts sont à la fois gérables et prévisibles (7). Une mise en garde s'impose toutefois : 17 de ces 19 pays sont des pays à revenu élevé, ce qui signifie que, dans l'ensemble, ils peuvent supporter les coûts et ont la capacité de gérer le programme.

De même, les difficultés liées à l'évaluation de la causalité semblent dépendre des ressources, car les pays qui ont adopté les programmes d'indemnisation des victimes d'une vaccination ont l'expertise nécessaire pour évaluer la qualité du vaccin et la causalité des blessures. Enfin, à ce jour, il n'y a pas suffisamment de données probantes pour évaluer la préoccupation concernant la possibilité de diminuer la confiance du public envers les vaccins. L'absence d'un programme d'indemnisation des victimes d'une vaccination n'a pas été considérée comme une préoccupation majeure par ceux qui hésitent. Rien ne prouve que les programmes d'indemnisation des victimes d'une vaccination appuient l'acceptation d'un vaccin; cependant, lorsqu'un programme d'indemnisation des victimes d'une vaccination est adopté, il pourrait y avoir une stratégie de communication qui rassure le public que, tout comme une assurance en cas d'accident, il sera couvert s'il y en a un.

\section{Conclusion}

Plusieurs pays riches ont des programmes d'indemnisation des victimes d'une vaccination; le Canada et les États-Unis sont les seuls pays du G7 qui n'en ont pas. La mise en œuvre des programmes d'indemnisation des victimes d'une vaccination se justifie fortement sur le plan de la santé publique. Bien qu'il n'y ait pas de preuve directe que ces programmes améliorent l'acceptation des vaccins, ils aident à maintenir l'approvisionnement en vaccins. Si d'autres provinces et territoires du Canada envisagent de tels programmes, les considérations éthiques, juridiques et pratiques ainsi que le bilan positif de 35 ans d'administration du programme au Québec peuvent aider à éclairer cette décision stratégique. 


\section{Déclaration des auteurs}

E. D. - Rédaction de la première ébauche et révision

D. G. - Rédaction et révision

N. E. M. - Rédaction et révision

S. H. E. H. - Rédaction et révision

S. H. - Rédaction et révision

\section{Conflits d'intérêts}

E. Dubé a reçu des subventions de l'Agence de la santé publique du Canada, du ministère de la Santé et des Services sociaux du Québec, des Fonds de recherche du Québec-Santé, des Instituts de recherche en santé du Canada, du Réseau canadien de recherche sur l'immunisation et du Conseil de recherches en sciences humaines du Canada. Elle est membre de l'équipe du Centre canadien de ressources et d'échange de données probantes sur la vaccination (CANVax).

N. E. MacDonald a reçu des subventions de l'Agence de la santé publique du Canada, de l'Organisation mondiale de la Santé, de la Nova Scotia Health Research Foundation, des Instituts de recherche en santé du Canada, du Réseau canadien de recherche sur l'immunisation et du Conseil de recherches en sciences humaines du Canada. Elle est membre de l'équipe CANVax.

\section{Remerciements}

La production des mémoires du Centre canadien de ressources et d'échange de données probantes sur la vaccination (CANVax) a été rendue possible grâce au financement de l'Agence de la santé publique du Canada. Merci aux nombreux auteurs, partenaires de vaccination et examinateurs qui contribuent à CANVax.

\section{Financement}

L'élaboration des mémoires du Centre canadien de ressources et d'échange de données probantes sur la vaccination est appuyée par le Fonds de partenariat d'immunisation de l'Agence de la santé publique du Canada.

\section{Références}

1. Looker $\mathrm{C}$, Kelly H. No-fault compensation following adverse events attributed to vaccination: a review of international programmes. Bull World Health Organ 2011;89(5):371-8. DOl PubMed

2. World Health Organization. Global Manual on Surveillance of Adverse Events Following Immunization. Geneva $(\mathrm{CH})$ : WHO; 2014 (modifié 2016; accédé 2019-05-15). https:// www.who.int/vaccine_safety/publications/Global_Manual_ revised_12102015.pdf?ua $=1$

3. Halabi SF, Omer SB. A Global Vaccine Injury Compensation System. JAMA 2017;317(5):471-2. DOI PubMed

4. Attwell K, Drislane S, Leask J. Mandatory vaccination and no fault vaccine injury compensation schemes: an identification of country-level policies. Vaccine 2019;37(21):2843-8. DOI PubMed

5. Hapuhennedige $S$, Nisbet $C$. A scoping review on vaccine injury compensation. Understanding Canadian Immunization Policy. Background Paper. Simcoe Muskoka District Health Unit. 2017. p. 40.

6. Wilson K, Keelan J. The case for a vaccine injury compensation program for Canada. Can J Public Health 2012 Mar-Apr;103(2):122-4. DOI PubMed

7. Adam, Abudu v. Ledesma-Cadhit et al., 2014 ONSC 5726; Batten v. Boehringer Ingelheim (Canada) Ltd., 2017 ONSC 53; Re Tluchak Estate, 2018 SKQB 311.

8. 2006 CanLII 41574 (ON SC).

9. (1983) 7 DLR (4th) 37 (QC CA), affirmed in Lapierre v. A.G. (Québec), [1985] 1 SCR 241.

10. Gouvernement du Québec. Programme d'indemnisation des victimes d'une vaccination. Gouvernement du Québec; (modifié 2019; accédé 2019-05-15). https://www.quebec.ca/ sante/conseils-et-prevention/vaccination/indemnisation-de s-victimes-vaccination/ 